Dept. for Speech, Music and Hearing Quarterly Progress and Status Report

\title{
Phonatory vibrations in singers. A critical review
}

Sundberg, J.

journal: STL-QPSR

volume: 32

number: 1

year: 1991

pages: $\quad 037-051$ 



\title{
PHONATORY VIBRATIONS IN SINGERS. A CRITICAL REVIEW*
}

\author{
Johan Sundberg
}

\begin{abstract}
Most Westem opera singers report feeling phonatory vibrations, i.e., vibrations caused by phonation, in different parts of their bodies while singing. As one perceives one's own voice quite differently from one's listeners, it has been hypothesized that phonatory vibrations may be useful as a feedback signal for the control of phonation. These vibrations have been examined in some articles which are reviewed. It is concluded that in singing at fundamental frequencies lower than about $350 \mathrm{~Hz}$ (pitch F4), the amplitudes of the vibrations in the chest wall may be easy to use as a feedback signal, as they reflect an aspect of phonation which is relevant to singers. However, vibrations in the face and the skull seem to vary considerably between different vowels and can, therefore, be assumed to be more difficult to use for controlling phonation which is basically independent of vowel quality.
\end{abstract}

\section{INTRODUCTION}

Voice is often assumed to be associated with vibrations. This association is implicitly manifested in terms of the prevalent voice terminology. For example, one of the many terms used for one of the female vocal registers is "chest register" or "chest voice". Similarly, most singers claim that it is necessary to "place" the tone in the forehead, the neck, the "mask", the nose. These expressions are hard to understand if they do not mean that the singers "feel" the tone in these areas. A plausible physical correlate of such sensations seems to be vibrations.

The idea is interesting that the placing of the tone is an essential criterion for an appropriate tone production. If both a necessary and sufficient criterion, the sensation of placement should occur only under certain conditions and always under these conditions. In other words, the phonatory vibrations should have a close and robust relation to phonation. What could this relation be?

Many singers and teachers of singing maintain that phonation should be independent of the vowel produced. At least it should not be entirely different for different vowels and pitches. Thus, according to this view, the singer can produce any vowel at any pitch, provided the tone is appropriately produced. Therefore, if the phonatory vibrations are strongly dependent of vowel and pitch, it is hard to realize how they can be used as a criterion of correct type of phonation. This suggests that the vibrations appropriate as a feedback control of phonation should be independent of vowel and pitch.

Vibrations possess certain advantages over other sensations available to the singer for the purpose of controlling phonation. By contrast to the auditory image of one's own voice, vibrations are unaffected by the acoustics of the room in which the singer is performing. A good portion of the sound that the singer hears has been travelling around in the room for a longer or shorter time before hitting the singer's own ear. This sound has been heavily affected by the acoustics of the room. Therefore, the sound that the singer perceives of his/her own voice sounds quite differently depending on the acoustic characteristics of the room. In a highly reverberant environment, it probably sounds quite brilliant while in a highly absorbing room, it may sound quite dull. The significance of the acoustic characteristics of the auditory feedback is manifested in terms of the common joy of singing in the bath tub, among other things.

On the other hand, the room acoustics does not have any influence on phonatory body vibrations. Therefore, these vibrations should be much more reliable as a feedback than the sound. There are also other reasons, to be mentioned later, why phonatory vibrations can be expected to have a great relevance for singers. In any event, it is an interesting question WHERE, HOW, and WHY singers

*This paper was presented at the Seminar Vibration and Contraction in Music Performance, arranged by the Music Acoustics Committee of the Royal Swedish Academy of Music in May 1990. 
vibrate while they sing. The present article attempts to answer these questions and, thus, to shed some light on the usefulness of such vibration sensations to the phonatory control needed in singing.

\section{ORIGIN OF PHONATORY VIBRATIONS}

The vocal fold vibrations alternately open and close the glottis just behind the Adam's apple. The frequency corresponds to the fundamental frequency of the tone sung. For example, if one sings the pitch of A4, the vocal folds meet and part 440 times per second, so the cycle time is $1 / 440=2.3 \mathrm{~ms}$. The closing is typically completed in something like a fifth of the period time, or $0.5 \mathrm{~ms}$ in this case (Sundberg, 1987). Assuming that the vocal-fold vibration amplitude is $1 \mathrm{~mm}$, we can estimate the fold's speed during closing to $2 \mathrm{~m} / \mathrm{s}$ in this case. It is apparent that such collisions must generate shock waves in the tissues, i.e., body tissue vibrations. Theoretically, these vocal fold collisions constitute one possible reason for phonatory vibrations.

However, the significance of these impacts from the vocal fold collisions to phonatory body vibrations seems rather small. Kirikae, Sato, Oshima, \& Nomoto (1964) excited the mouth cavity at $250 \mathrm{~Hz}$ by means of a tube attached to a loudspeaker and by means of vibrator attached to the upper incisors. They found much lower vibration amplitude on the skull from the acoustic excitation than from the mechanical vibration; however, they did not control for the amplitude of the excitation in the experiment.

In an experiment, the author brought the thyroid cartilage to vibration by means of a vibrator (Sundberg, 1983). The amplitude of the shaker vibrations was adjusted so that thyroid vibrations which were of the same magnitude as those typically occurring during phonation were generated. Simultaneously, the concomitant vibration level was measured on the tracheal wall just above the clavicles and on the sternum. The results showed that these vibrations, which thus had a purely mechanical origin, generated trachea and sternum vibrations which were about $20 \mathrm{~dB}$ below those caused by phonation. This shows that the sternum vibrations cannot be generated by the impact impulses caused by the vocal fold collisions. This result was later corroborated by Sonninen, Vilkman, Karppinen, \& Karppinen (1986).

Another possible source of the phonatory body vibrations are the sound pressure oscillations within the phonatory apparatus. The background is the following. When the vocal folds alternately open and close the passage for the airstream in the trachea, vigorous pressure variations are generated within the vocal tract, i.e., in the pharynx and the mouth. According to Fant's (1960) calculations, the sound pressure level in the vocal tract is in the vicinity of $130 \mathrm{~dB}$, or $10 \mathrm{~dB}$ above the threshold of pain.

These pressure variations can be recorded directly in the vocal tract and trachea (Kitzing \& Löfqvist, 1975). One uses minute pressure transducers adapted on a very thin wire, as shown in Fig. 1. Miller \& Schutte (1984) had a bass-baritone subject inhale such a device. The position of the wire was adjusted so that one transducer was just above the vocal folds and the other just below them, as shown in the same figure. They found that the sound pressure varied on the order of magnitude of $+/-1 \mathrm{kPa}\left(10 \mathrm{~cm} \mathrm{H}_{2} \mathrm{O}\right)$. These values are similar to those found by Kitzing \& Löfquist (1975) in a male subject with a normal speaking voice. This pressure amplitude corresponds to a sound pressure level of $94 \mathrm{~dB}$, thus somewhat lower than Fant's estimate. Later, Schutte \& Miller (1986) repeated the experiment with a soprano and found considerably greater pressure variations at the pitch of B5 $(960 \mathrm{~Hz})$. Here the amplitude was close to ca $4 \mathrm{kPa}$ corresponding to a SPL of 106 dB.

These pressure variations deserve a closer examination. Fig. 2 compares pressure signals recorded while the subject was singing different vowels at the same pitch of $\mathrm{C} 4(262 \mathrm{~Hz})$. The figure shows that the pressure signal above the vocal folds depends very strongly on the vowel, while below the folds only minor differences can be observed between different vowels. Here, all vowels generated a signal characterized by a big deflection followed by a smaller one.

This vowel dependence is not surprising. The differentiation between vowels happens supraglottally, while the signals represented by the pulsating transglottal airflow is basically the same for all vowels. The trachea normally keeps a constant shape and, therefore, its resonance characteristics remain basically unchanged for all vowels. Thus, it should always respond in the same way to the sound generated subglottally by the pulsating transglottal airflow. Consequently, one should expect 
the pressure variations in the trachea to be determined by the vibratory pattern of the vocal folds or, more exactly, the pulsating transglottal airflow.

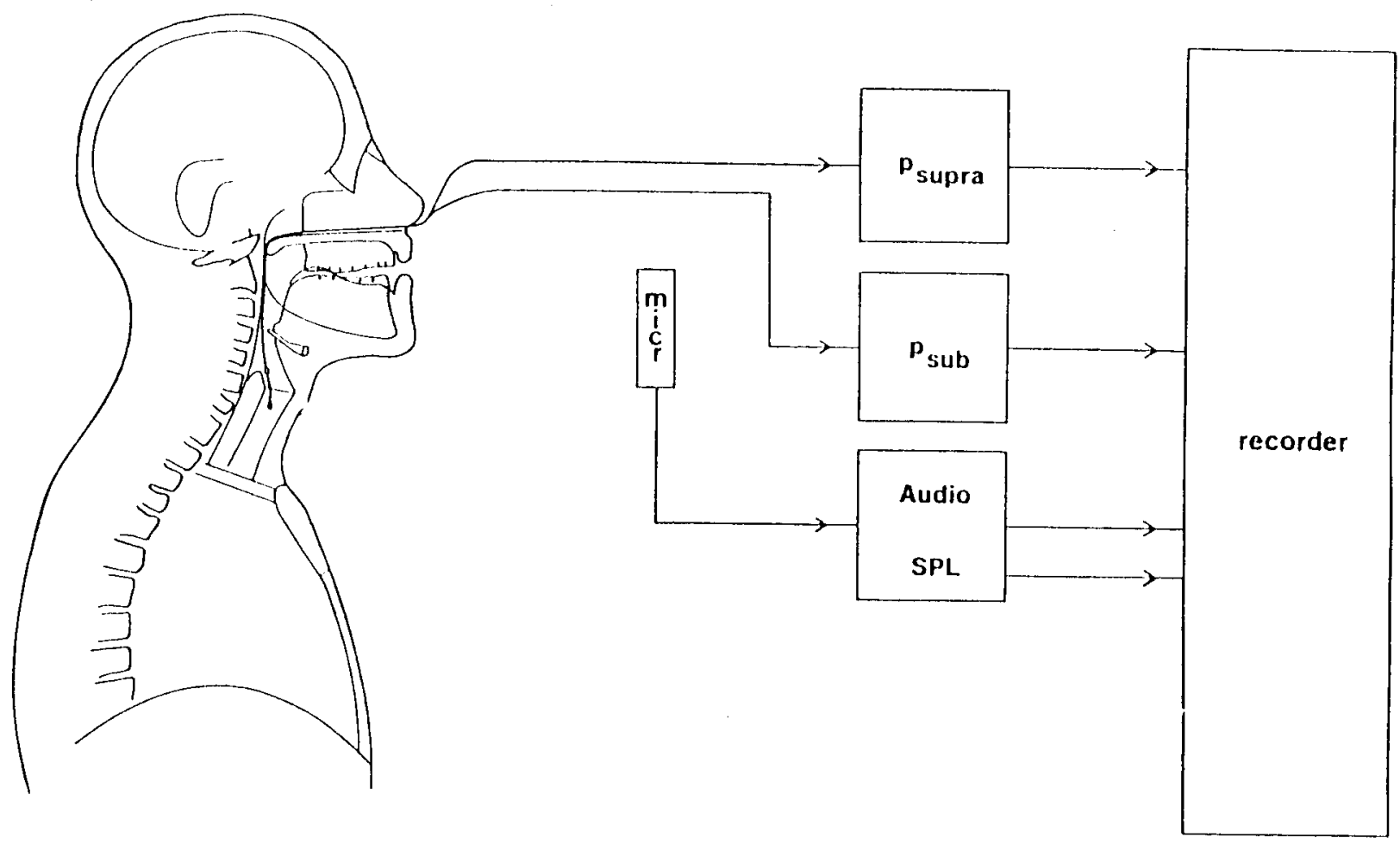

Fig. 1. Schematic illustration of the technique used by Kitzing \& Löfqvist (1975) and by Miller \& Schutte (1984) for recording the sound pressure oscillations below and above the vibrating vocal folds (psub and psupra). The signals are picked up by minute pressure transducers adapted on a very thin wire and placed in the sub-and supraglottal airways, respectively. A microphone (micr) picks up the audio signal providing SPL data. All signals are recorded on a tape recorder. (From Miller \& Schutte, 1984).

Sufficiently loud sounds in an enclosure obviously bring walls to vibrate. The very strong sound pressures in the vocal tract and in the trachea cause adjacent tissues to vibrate. This is the origin of the phonatory vibrations in the skull, neck, and chest regions.

Fig. 3 illustrates how the pressure variations above and below the glottis vary with pitch. The pitch dependence is quite strong in both locations. For the pitch F3 (approximately $175 \mathrm{~Hz}$ ) the waveform above the glottis shows a greater and a smaller peak followed by a valley, but one octave higher, at F4, the secondary peak has entirely disappeared, and the waveform rather looks like a sinewave. The waveform below the glottis varies somewhat less. At the pitch F3, it looks like a decaying sinewave with three peaks of decreasing amplitudes and one octave higher, there is not enough time between the periods for more than two such peaks.

\section{VIBRATION MEASUREMENT}

Phonatory vibrations are easy to pick up and record. This can be realized by means of certain microphones, so called accelerometers. These are totally insensitive to sound and react only to vibrations. Vibrations can be recorded in terms of the displacement of a body such as a wall. The unit is then given in a distance unit such as $\mathrm{mm}$. Vibrations can also be measured in terms of the velocity of the body with the unit $\mathrm{m} / \mathrm{s}$. A third possibility is to measure the acceleration, i.e., the change in velocity with the unit $\mathrm{m} / \mathrm{s}^{2}$. The acceleration is the time derivative of the velocity which in turn, is the time derivative of the displacement. A derivation of a complex tone corresponds to a boosting of $6 \mathrm{~dB}$ /octave of the spectrum. Consequently, the acceleration and velocity spectra slope 12 and 6 $\mathrm{dB} /$ octave less steeply than the displacement spectrum, respectively. In other words, the sensitivity to high vibration frequencies is greatest if measured in terms of acceleration and smallest if measured in terms of displacement. 
PIITCH CA $(262 \mathrm{H} \%)$

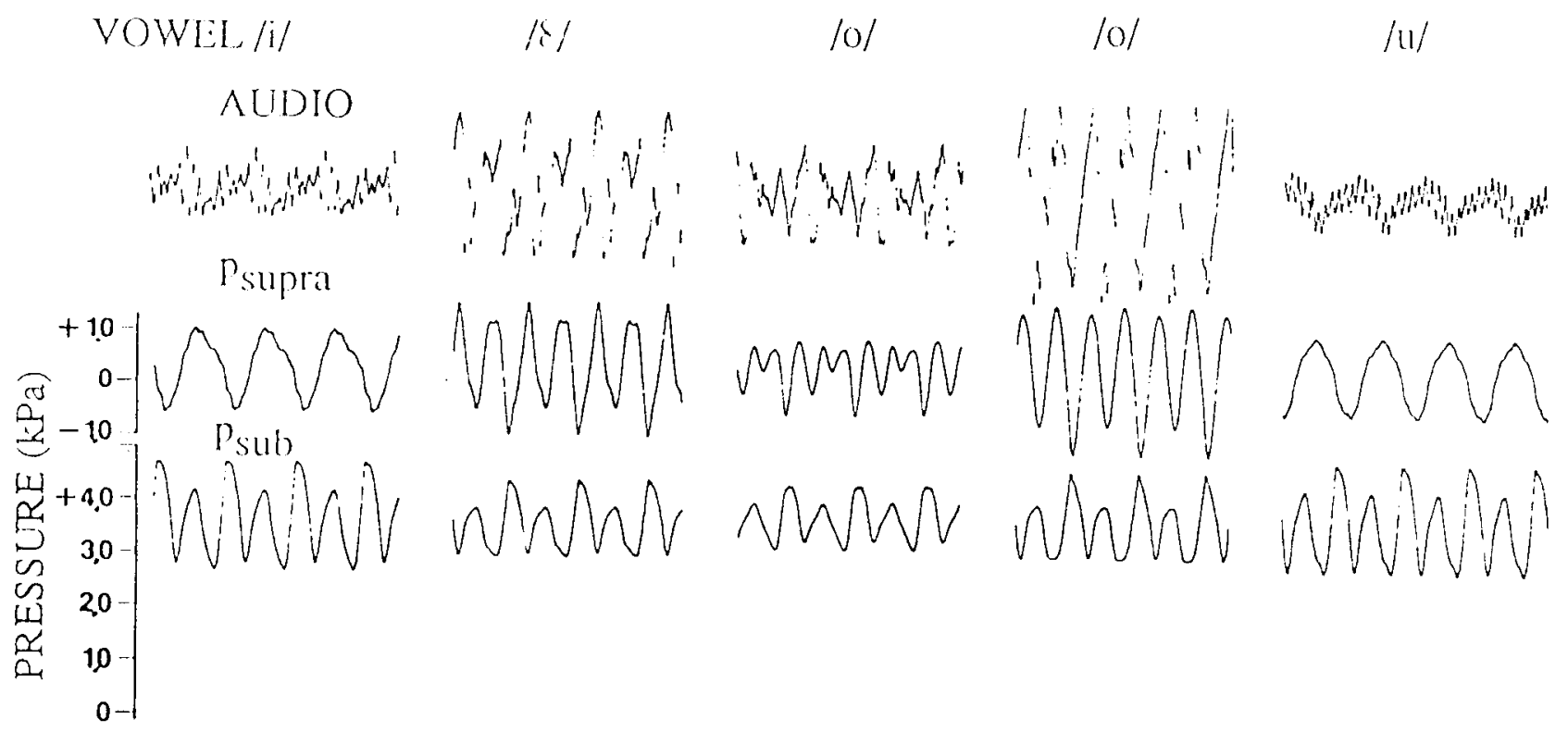

Fig. 2. Audio signal and sub-and supraglottal pressure oscillations (psub and psupra) recorded while the subject was singing the indicated vowels at $262 \mathrm{~Hz}$ fundamental frequency. (From Schutte \& Miller, 1986).

VOWEL/i/

PITCH F3

$\wedge 3$

D4

F4

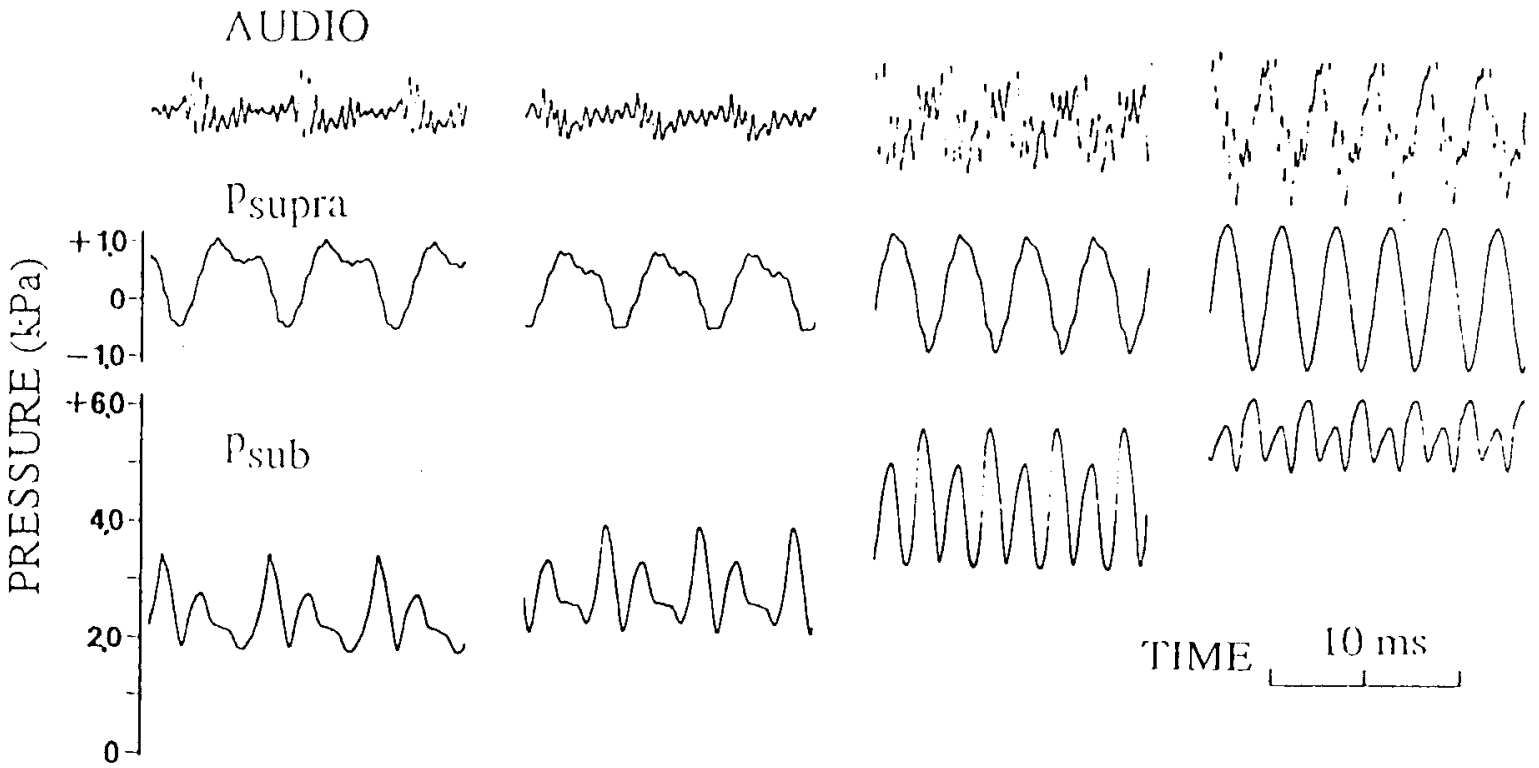

Fig. 3. Audio signal and sub-and supraglottal pressure oscillations (psub and psupra) recorded while the subject was singing the vowel lil at the indicated pitches. (From Schutte \& Miller, 1986).

\section{INPUT IMPEDANCE}

How willing to vibrate are the face and the throat? This can be expressed in terms of the input impedance, defined as the ratio between the driving force and the resulting displacement. In most systems the input impedance varies with frequency, culminating at certain frequencies called the resonance frequencies. Below the lowest resonance frequency, the frequency dependence is reasonably simple.

Fant, Branderud, \& Nord (1976) measured the input impedance of the vocal tract walls at low frequencies, far below the lowest vocal tract resonance frequency.

They inserted a small plastic tube attached to a loudspeaker element in their male subject's mouth 
and sent a low frequency sinewave into the vocal tract. The vibrations were recorded by means of an accelerometer which they fastened to various places on the face and the neck.

Fig. 4 shows the results. The contours show the distribution of equal amplitude in approximately the same way as altitudes are represented in a topographic map. The figure shows that the closed lips vibrated at the greatest amplitude. This is natural in view of the fact that these tissues are comparatively thin. The profile also shows the larynx region. There, the vibration amplitude was maximum, a few $\mathrm{dB}$ greater than for the lip region. The amplitude decreases with the distance to the larynx and lip regions. These measurements reveal that at very low frequencies, the willingness of the vocal tract walls to vibrate is greatest near the vocal tract ends, the lips, and the larynx.

\section{PHONATORY VIBRATIONS}

Using laser technique, phonatory vibrations can be recorded by means of photography. The displacement amplitude then appears in the same way as altitude is represented in a topographical map, similar to what was shown in Fig. 4. The higher the number of contours encircling a region, the greater the vibration amplitude in that region. This technique was used by the Polish researcher Pawlowski and co-workers (Pawlowski, Pawloczyk, \& Kraska, 1985), and by Sonninen and associates (Sonninen \& al., 1986).

Fig. 5 shows some examples of such recordings for a soprano singing the vowel /a/ at different pitches. It can be observed that the larynx vibrates rather vigorously for the low tone while the greatest amplitudes appear in the lip region for the highest pitch. Fig. 6 shows the corresponding data for a bass singer. The larynx vibrations are much greater than for the soprano and somewhat smaller for the highest pitch than for the lower one. Apparently, the larynx vibrates more willingly at low frequencies. For each of the singers, one can also observe a tendency for high pitches to produce smaller displacement amplitudes than for low pitches.

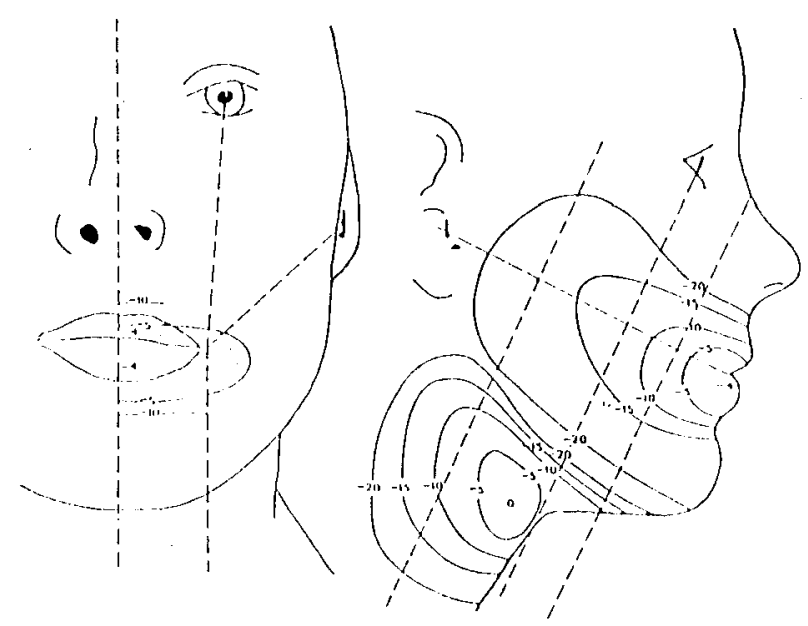

Fig. 4. Equal-vibration-level contours as measured when the vocal tract walls were vibrated by means of a low frequency sine tone induced through a thin tube which the subjects held in his mouth corner. Vibration levels are given in $d B$ re the level recorded on the thyroid cartilage. (After Fant \& al., 1976)

As was mentioned before, the most common method of measuring vibrations is to use an accelerometer. An early accelerometer investigation of phonatory vibrations was carried out by Kirikae and co-workers (Kirikae \& al., 1964). They fastened accelerometers on the skull, the forehead, the cheek, the lower mandible, the larynx, and the sternum. The subjects pronounced five different vowels.

Fig. 7 shows the vibration levels measured. It can be observed that the vibration level varies considerably depending on the vowel. On the forehead, the cheek, and lower mandible the vibrations are greatest on the vowels $/ \mathrm{i} /$ and $/ \mathrm{u} /$, lowest for $/ \mathrm{a} /$, while $/ \mathrm{e} / \mathrm{and} / \mathrm{o} /$ give intermediate values. This vowel dependence is not surprising as the sound pressure oscillations within the vocal tract are quite vowel dependent, according to the measurements mentioned above. The vibrations are greatest on the larynx which is in good agreement with Pawlowski's laser photographs just discussed. Also on the sternum the vibrations are vigorous. Here, the amplitude is not very vowel dependent. This is in accordance with the previous observation that the pressure variations in the trachea are rather independent of the vowel. 

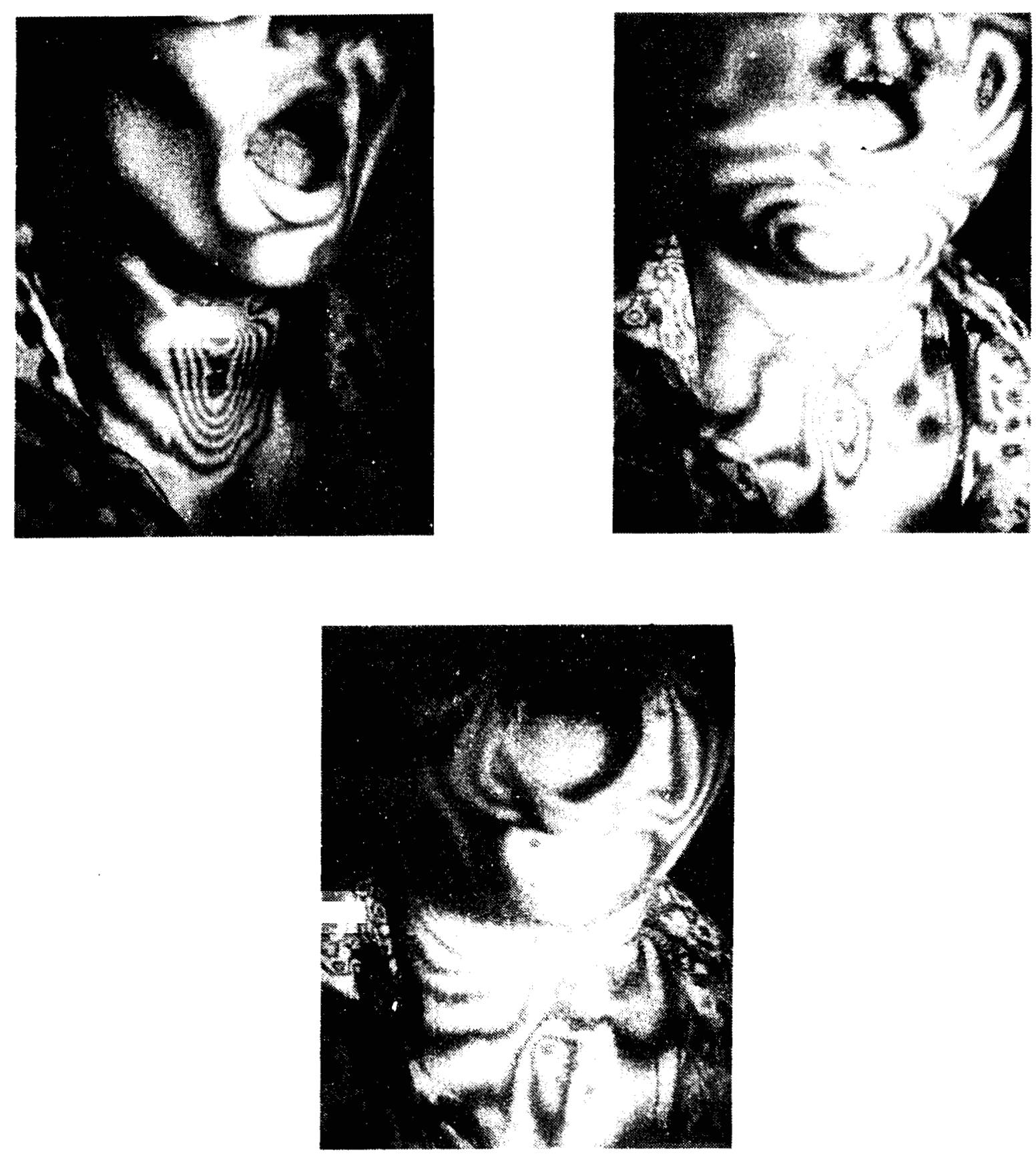

Fig. 5. Phonatory vibrations as recorded by means of laser technique. The patterns show equal-vibration-amplitude contours for a soprano singing the vowel /al at the pitches indicated. (After Pawlowski \& al., 1985). 

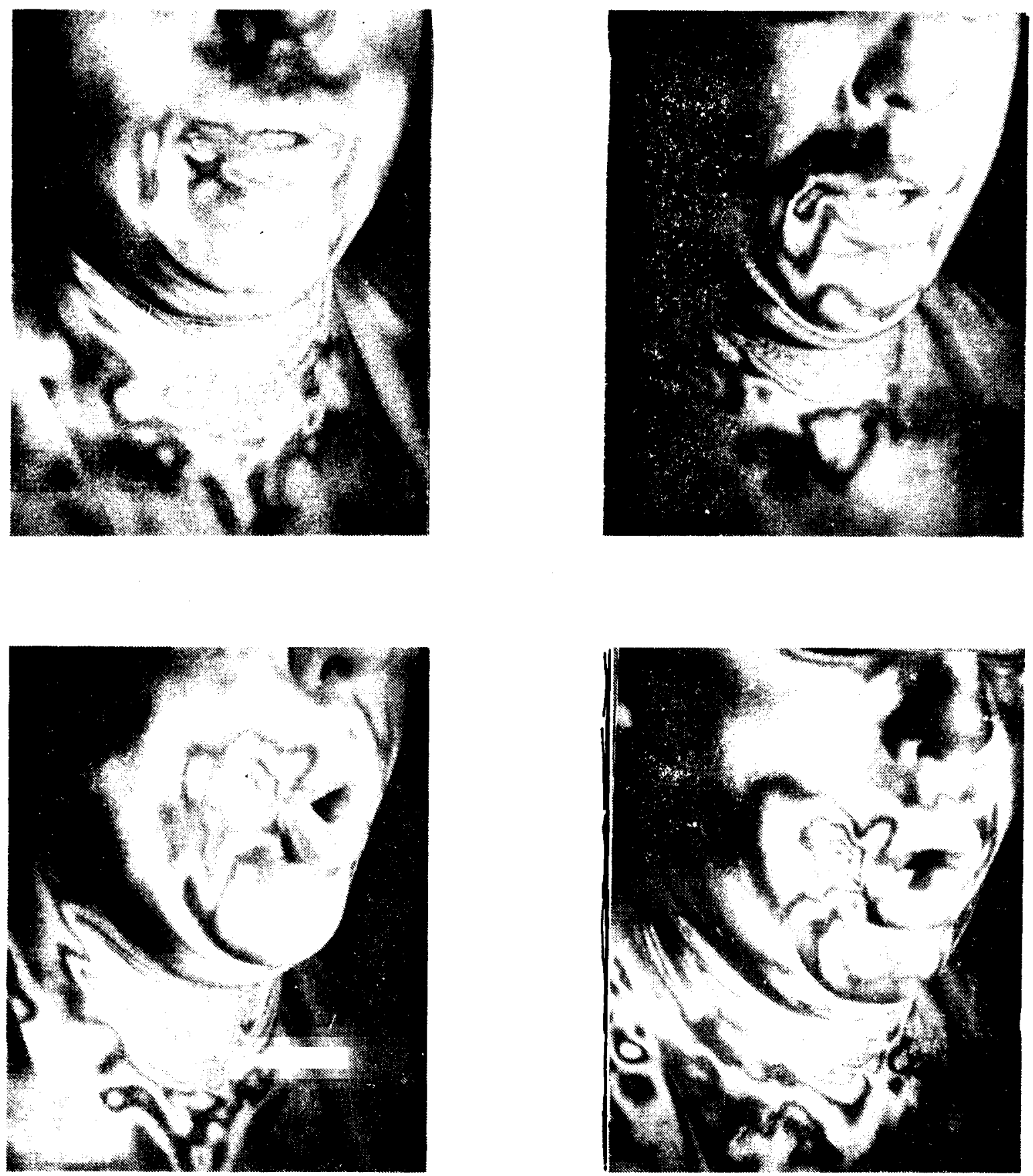

Fig. 6. Phonatory vibrations as recorded by means of laser technique. The patterns show equal-amplitude-vibration contours for a bass singer singing the indicated vowels at the given pitches. (After Pawlowski \& al., 1985). 
Kirikae and co-workers demonstrated that the vibration level on the skull varied systematically with the lip opening: the wider the opening, the smaller the vibration level. This phenomenon can easily be verified simply by putting one's index finger on the top of the skull while pronouncing alternately the vowels /u/ and /a/; the vibrations can be clearly felt only for $/ \mathrm{u} /$. This result suggests an explanation why different vowels generate vibrations of different amplitudes in locations above the glottis. The background is the following.

Vowels differ acoustically with regard to the vocal-tract resonances, the so called formants. Formants are manifested in terms of peaks in the frequency curve of the vocal tract. Their frequencies, the formant frequencies, are determined by the shape of the vocal tract, also including the termination represented by the lip opening. When the lip opening is widened, the first formant frequency is raised, other factors being equal. The frequencies of the formants are reflected in the sound pressure oscillations within the vocal tract. Therefore, the influence of the lip opening on the skull vibrations would merely reflect a dependence on the sound-pressure oscillations and, hence, on the first-formant frequency: the lower the first-formant frequency, the greater the skull vibrations.

These assumed relations between the first formant frequency and the phonatory skull vibrations seem to apply to vibrations also in other locations. It was mentioned that the vowels $/ \mathrm{i} / \mathrm{and} / \mathrm{u} / \mathrm{gave}$ stronger vibrations on the forehead, cheeks and lower mandible, according to the Kirikae investigation. These vowels have the lowest first-formant frequency value among the vowels tested, and /a/, showing the lowest vibration level, has the highest first-formant frequency.

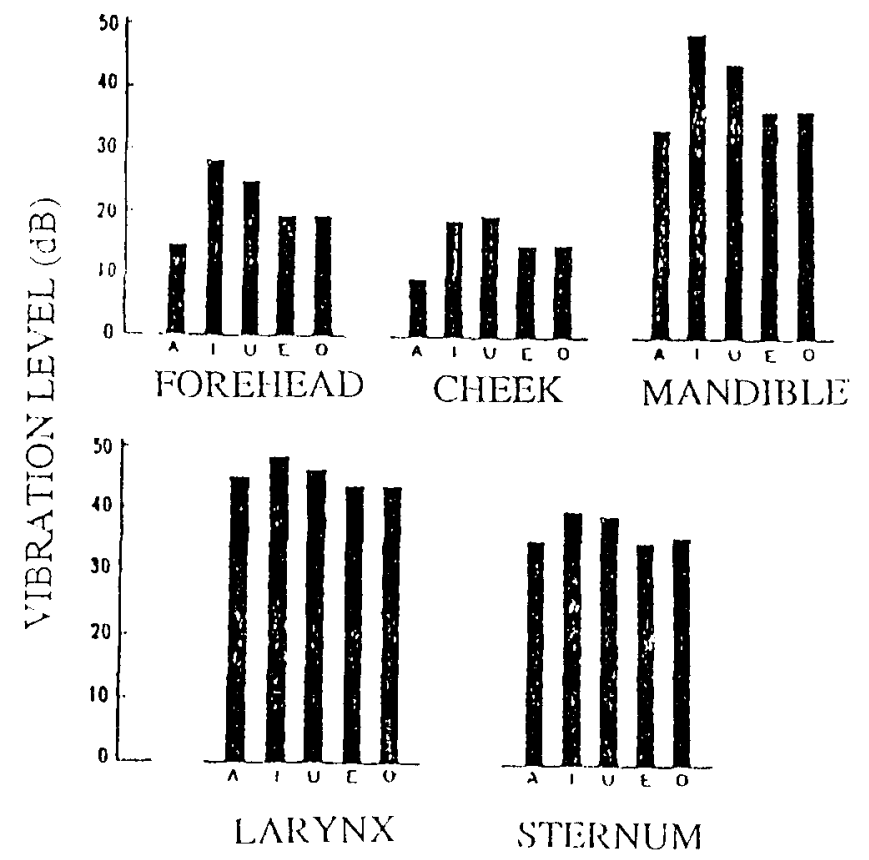

Fig.7. Vibration levels as measured by means of accelerometers in the indicated locations while a male subject pronounced different vowels at $125 \mathrm{~Hz}$ fundamental frequency. In each panel, the bars, counted from left to right refer to the following vowels: $|a|,|i|, \mid$ ul, $|e|, \mid$ ol. (After Kirikae \& al., 1964).

According to the Kirikae investigation, the sternum vibrations were strongly dependent on register, as shown in Fig. 8. Thus, they found that singing in chest register gave considerably higher amplitudes than singing in head register. However, the tones compared differed not only with regard to register but also with regard to pitch. As we know already from the investigation by Pawlowski \& al. (1985), fundamental frequency has an effect on vibration amplitude. Therefore, the relation between register and sternum vibrations need not necessarily be as described in the Kirikae study.

Milutinovic (1988a; 1988b) fastened accelerometers to thirteen different locations and measured the overall level of the vibration signal. His findings were in agreement with those gained by Fant and co-workers in the low frequency range; the thyroid vibrations are far more powerful than vibrations in adjacent structures. In more remote structures, such as at on the spine the projection of the seventh thoracic vertebra, the vibration amplitude was found to be very small indeed, $-30 \mathrm{~dB}$ below the amplitude of the thyroid membrane. Also, the overall level was found to vary considerably with vowel. 
Milutinovic also measured the spectrum of these vibrations. The bandwidth of the spectrum varied greatly with position. He found heavily low-pass filtered signals on the spine while, in the area of the nasal bone, partials up to $1 \mathrm{kHz}$ and more were measured. The type of voice used was another factor of significance to the vibration spectrum. A clearly nonperiodic vibration signal dominated by low-frequency components was observed in the case of a patient suffering from hyperkinesia.

Given Milutinovic's finding that the vibration amplitude decreases considerably with the distance to the thyroid cartilage, it is surprising that Frei (1978) could record vibrations also in a plate on which a singer subject was standing. Moreover, different vibration waveforms were observed for different vowels and types of voice; yet we have seen above that below the thyroid region, the vowel dependence of the phonatory vibrations is small. Frei expressed an optimistic view as to the usefulness of these vibrations as a feedback for phonatory control.

Milutinovic found that the attenuation of phonatory vibration from the thyroid to the spine was 25 $\mathrm{dB}$, approximately. Further, according to Rasmussen (1982), the vibration attenuation between the chest region and the feet is close to $20 \mathrm{~dB}$. This would mean that the total attenuation of vibrations between the thyroid and the feet is $45 \mathrm{~dB}$, approximately. It seems likely that the sound wave in the recording studio would cause a more significant vibration of a foot plate of the size used by Frei than the vibrations transferred mechanically from the phonatory apparatus.

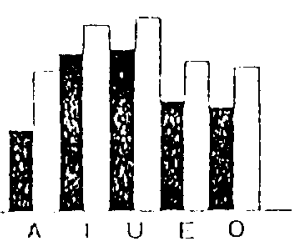

SKULL

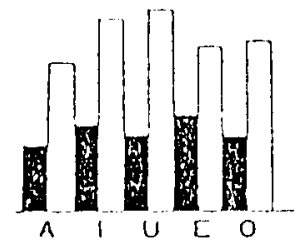

NASALDORSUM

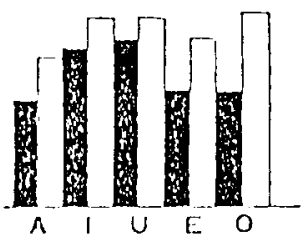

MANDIBLE
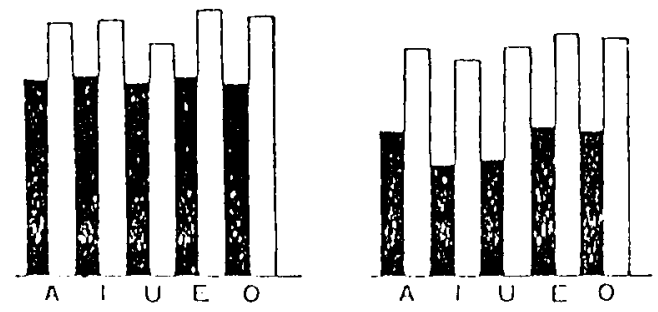

"CHISTT VOICL"

"III:AD VOICI:"

STLARYNX D

\section{BRI STERNUM TTA}

Fig. 8. Vibration levels measured by means of accelerometers in the indicated locations while the subject, an alto singer, sang different vowels in chest and head register at 250 and $500 \mathrm{~Hz}$ fundamental frequency, respectively. In each panel, the bars, counted from left to right refer to the following vowels: $|a|,|l|,|\mathrm{u}|,|\mathrm{e}|, \mid \mathrm{o} /$. (After Kirikae \& al., 1964).

Recently, Sakakura \& Takahashi (1988) returned to the same question as Kirikae and co-workers. They used seven subjects, male and female amateur singers. Accelerometers were fastened to the forehead, cheek, clavicle, and sternum. Also they recorded the sound in front of the mouth. Their measurements concerned acceleration. As Milutinovic (1988a; 1988b), they studied not only the overall amplitude but also the spectrum of the vibrations.

Fig. 9 shows the spectrum of the signals recorded for the vowel /a/ sung at a fundamental frequency of $314 \mathrm{~Hz}$. The partials below about $1000 \mathrm{~Hz}$ can be identified in all spectra. Some accelerometer locations give a less clear overtone structure than others. The fundamental is dominating on the clavicles and the sternum, less so for the forehead and the cheek. On the forehead, on the clavicle and on the sternum, one can observe a group of prominent partials near $3 \mathrm{kHz}$, as indicated by the arrow in the figure. These partials correspond to the singer's formant, a group of adjacent partials with particularly high amplitudes which belong to the main spectrum characteristics of voiced sounds sung by male singers and altos. Nonsinger subjects lacked this characteristic in their vibration spectra.

The vibration amplitude obviously depends on how loud one sings, and different subjects do not sing equally loud. We have also seen that for locations above the glottis, the vowel is significant to 
the vibration amplitude. Hence, there are good reasons to relate the vibration amplitude to the amplitude of the sound produced. Henceforth, we will refer to this ratio as the normalized vibration level.

Sakakura \& Takahashi (1988) found that the normalized vibration level varied considerably with vowel, voice type, register, and location. For example, the nonsingers showed weaker normalized vibration level than the singers, particularly for the vowel /a/, but there were substantial individual differences both within the singers' and the nonsingers' groups. The vowel /a/ generated weaker normalized vibration levels than the vowel /i/. On the other hand, the different registers did not show any consistent differences. In this regard, the results did not support the observation made by Kirikae \& al. that register had a great effect.

Sakakura \& Takahashi found a striking difference between some of the singers and the nonsingers, as illustrated in Fig. 10. It shows the average of the normalized vibration level for the three singing student sopranos when they sang and used their "untrained voice", respectively. It can be observed that for the vowels $/ \mathrm{i} /, \mathrm{u} /$, and /e/, the normalized vibration level in the cheek was considerably greater in singing. Similar findings were made for tenors, but not for baritone and bass singers.

\section{VIBRATION SPECTRA}

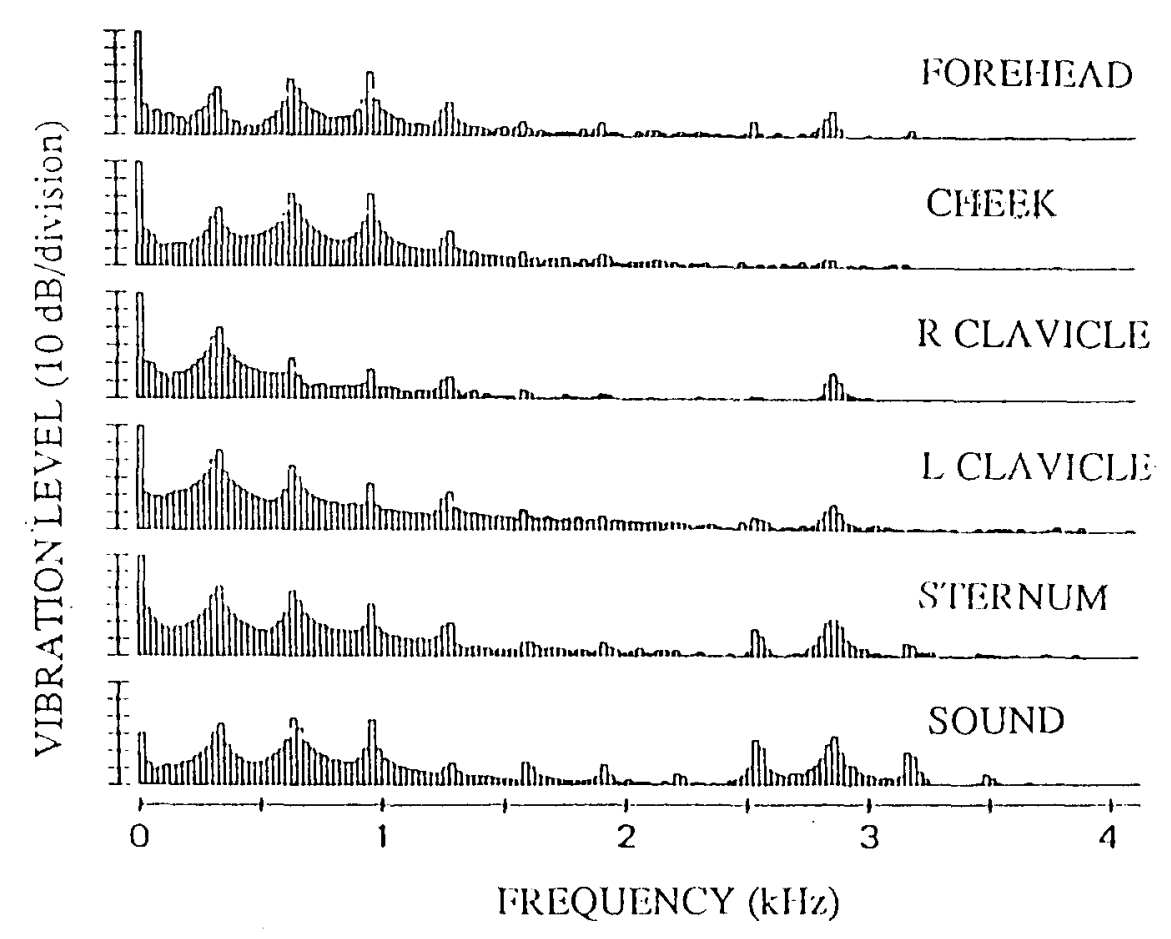

Fig. 9. Spectra of vibration signals recorded by accelerometers in the indicated locations for the vowel lal sung by a female singer using her trained voice at $314 \mathrm{~Hz}$ fundamental frequency. (From Sakakura \& Takahashi, 1988)

Unfortunately, these results are somewhat problematic. The confounding factor is that the normalized vibration level compares the overall level of signals which may differ considerably with regard to the spectrum. The overall level of a complex tone, such as a vowel, is mostly determined by the levels of a quite small number of partials, viz. the very loudest ones (Gramming \& Sundberg, 1988). This holds for both sound spectra and vibration spectra.

In a vowel spectrum where the first formant frequency is well above the fundamental, an overtone is normally the loudest partial; in such cases, a level increase of a few $\mathrm{dB}$ of the fundamental does not necessarily lead to any appreciable increase of the overall sound level, other things being equal. In vowel spectra with the fundamental close to the first formant, on the other hand, the fundamental is the loudest partial. In such a spectrum, an increase of the level of the fundamental by a certain number of $\mathrm{dB}$ will lead to an increase of the overall sound level by a similar amount of $\mathrm{dB}$. The 
vowels for which increased cheek vibrations were noted were those which had the lowest first formant. In the spectra of the vibration signals for the vowels $/ \mathrm{i} /$ and $/ \mathrm{u} /$, the fundamental and the second partial were of similar levels. This means that the normalized vibration level is a rather complex measure; depending on what vowel and pitch is considered, it compares the sound levels of the same or different spectrum partials. Therefore, the exact significance of the results of Sakakura \& Takahashi regarding normalized vibration level is hard to realize.

\section{SINGING}

UNTRAINED VOICE
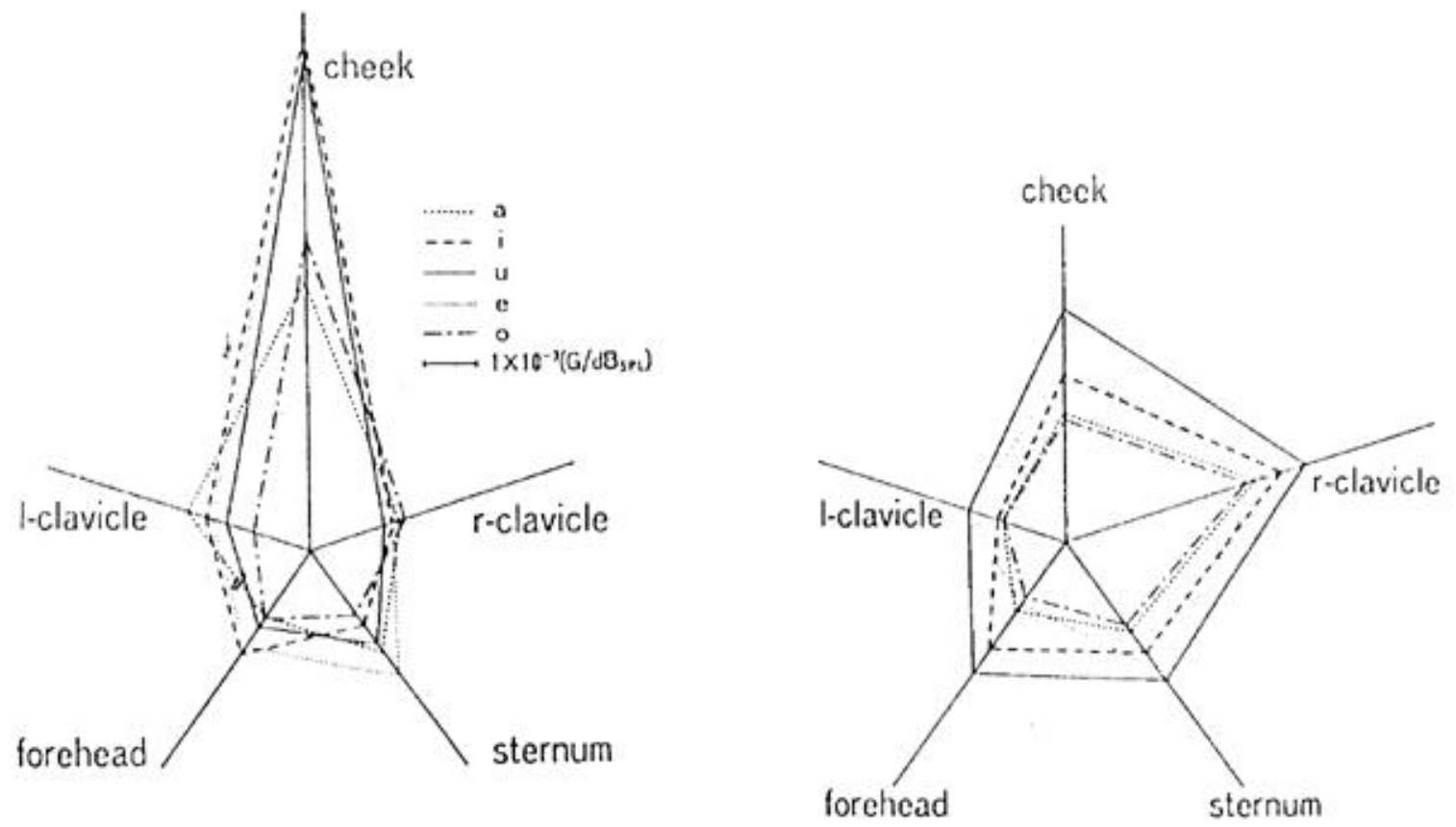

Fig. 10. Averaged normalized vibration level on the cheek for 3 soprano subjects when they used their singing voice (left) and their normal speaking voice (right). (From Sakakura \& Takahashi, 1988)

Summarizing, these results seem to demonstrate that the phonatory vibrations are greatly dependent on the vowel when recorded in locations above the glottis. This should make these vibrations hard for singers to use as feedback signal for controlling phonation. The vibrations that showed the smallest dependence of vowel were found below the glottis, on the trachea, the clavicles, and the sternum in both the Kirikae and the Sakakura investigations. The small effect of vowel in these locations agrees with the observation mentioned above that the pressure oscillations within the trachea show a very small vowel dependence. It seems that the vibrations appearing below the glottis should be easier to use for the control of phonation.

\section{CHEST WALL VIBRATIONS}

Phonatory chest wall vibrations were studied in detail for singers by the author (Sundberg, 1983). The vibrations were recorded on the sternum by means of an accelerometer and for comparison, vibrations on other locations as well as the sound were also examined. The subjects were seven male and female professional and amateur singers.

The distribution of vibration amplitude on the front part of the rib cage is illustrated in terms of average levels of a male subject in Fig. 11. The level recorded on the thyroid cartilage was used as the reference. The figure shows that the sternum vibrations had the highest level; as we have seen above, this observation was corroborated by Milutinovic.

Spectrum analysis was carried out for the vibrations of the thyroid cartilage, on the trachea at the level of the clavicles, and of the sternum. Three male singers served as subjects. As an average across vowels, the larynx vibrations turned out to contain the strongest overtones, while the overtones were always much weaker in the sternum vibrations. When measured in terms of displacement, the fundamental was almost invariably the strongest partial on the sternum for all subjects, pitches, and vowels. This is in good agreement with the observations by Sakakura \& Takahashi (1988). 
The sternum vibrations turned out to vary considerably with fundamental frequency, as illustrated in Fig. 12. The overall displacement level of the sternum decreased with fundamental frequency by an average of $9 \mathrm{~dB} /$ octave for the singers when they sang scales.

This last-mentioned observation is quite important as it reveals a complication in amplitude comparisons of signals differing in fundamental frequency; the fundamental frequency difference will automatically induce a difference in the amplitude of vibration. This may be the reason for the great amplitude difference which Kirikae and co-workers found when they compared a tone sung in chest register at $125 \mathrm{~Hz}$ with a tone sung in head register at $500 \mathrm{~Hz}$. At comparable fundamental frequency, the sternum vibrations in head register may very well be equal to or even stronger than those in chest register.

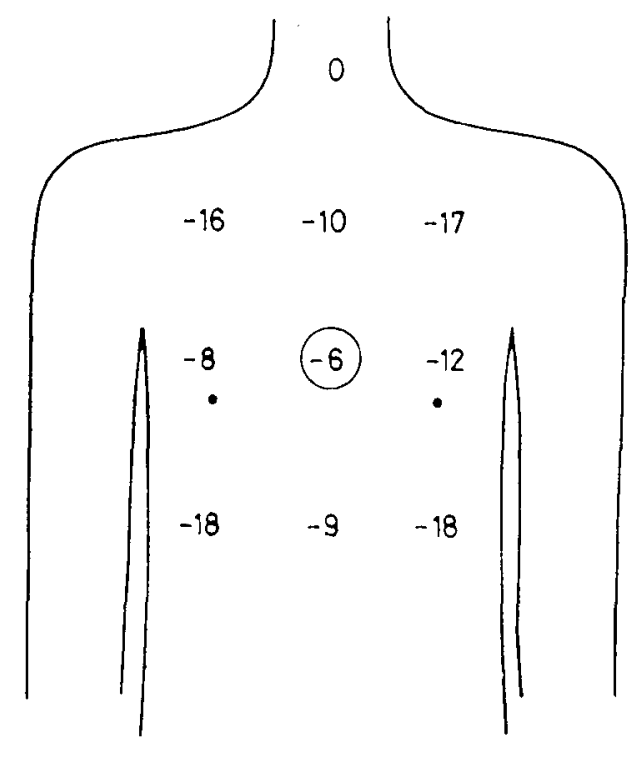

Fig. 11. Typical mean displacement levels for different locations on the chest wall of a male subject. (After Sundberg, 1983)

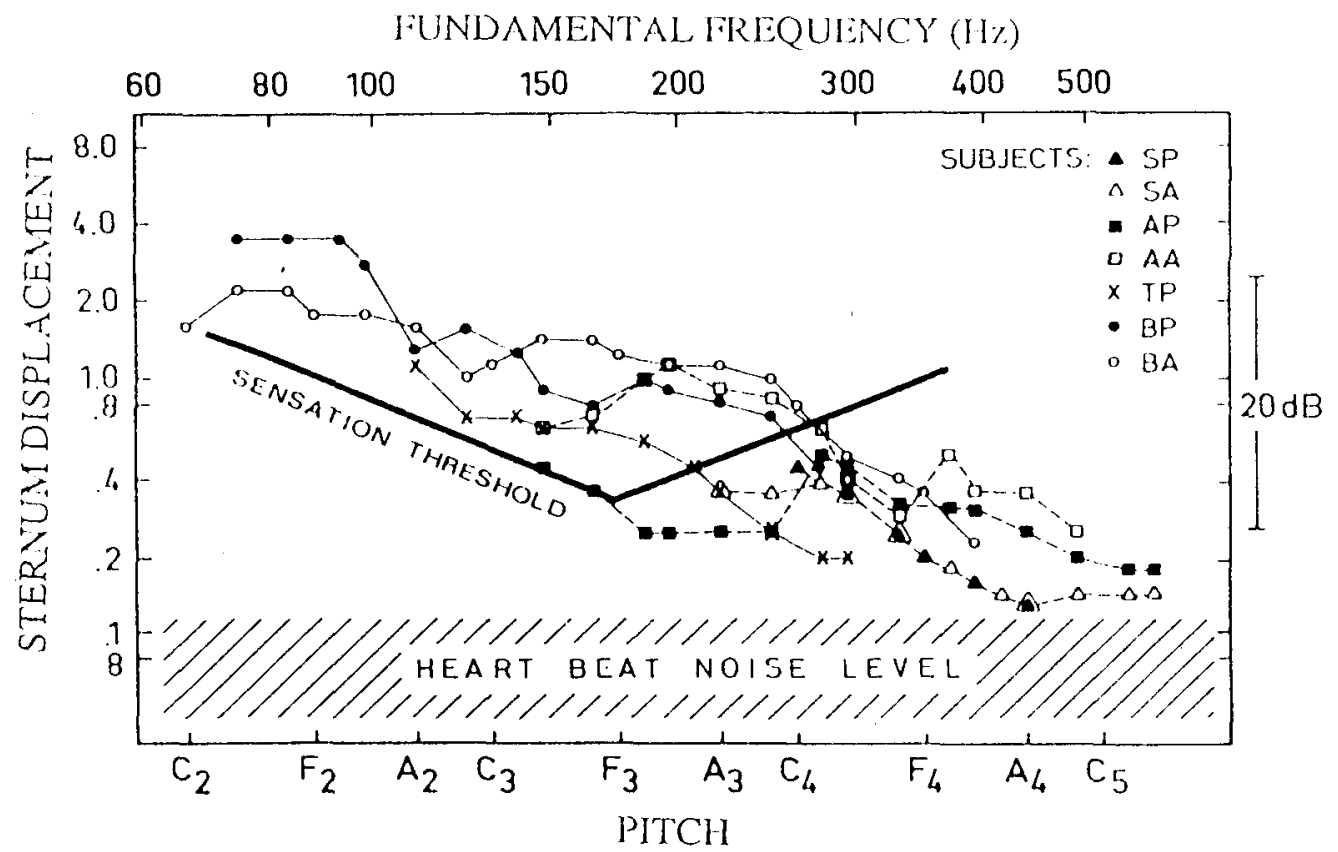

Fig. 12. Averaged sternum displacement levels as function of fundamental frequency for 7 singer subjects who sang scales throughout their pitch ranges. (From Sundberg, 1983)

Another interesting question is of course what voice property is reflected in terms of these vibrations. This question is obviously crucial for the usefulness of these vibrations as a feedback for the control of phonation. Measurements showed that the sternum vibrations were closely related to the 
level of the fundamental of the spectrum of the voice source, i.e., the pulsating airflow signal which is generated by the vocal-fold vibrations. This is illustrated in Fig. 13. The stronger the fundamental, the stronger the sternum vibrations. This covariation was observed in all singer subjects. The correlation coefficient for the level of the fundamental and the sternum vibration amplitude amounted to 0.89 , as a mean over subjects. This means that about $80 \%$ of the variations which occur in sternum vibration level reflect variations in the level of the fundamental.

Is this useful information for singers? To answer this question, we need to digress and consider the acoustic characteristics of the voice source. As mentioned, the vocal-fold vibrations chop the airflow from the lungs so that it is converted to a pulsating flow, the voice source. In analogy with almost all other sounds generated by traditional musical instruments, the human voice source consists of a fundamental and a series of harmonic overtones, the amplitudes of which decrease with their frequency. The loudness of phonation is controlled by means of the air pressure in the lungs. But the amplitude of the voice-source fundamental can be independently controlled irrespective of loudness. Two tones with exactly the same sound level may, therefore, differ considerably with regard to the level of the fundamental. This is true as long as the fundamental has a negligible influence on the sound level of the vowel, i.e., when the first-formant frequency is well above the fundamental frequency. This case is normal in speech and also in singing, except for the higher notes of tenors and female singers.

The variation of the amplitude of the fundamental is realized by the muscular force by which the vocal folds are adducted, i.e., pressed against each other. If this adduction force is too small, the folds fail to reach contact. Then, the fundamental is totally dominating the spectrum, and the voice sounds very soft, breathy, and leaking. If, on the other hand, adduction force is strong, i.e., the vocal folds are pressed forcefully against each other, the fundamental looses its dominating position in the spectrum, and the voice timbre becomes pressed, strained, or strangled. In case pitch and loudness are high, the voice sounds as a cry, a sound rarely regarded as a merit in singing.

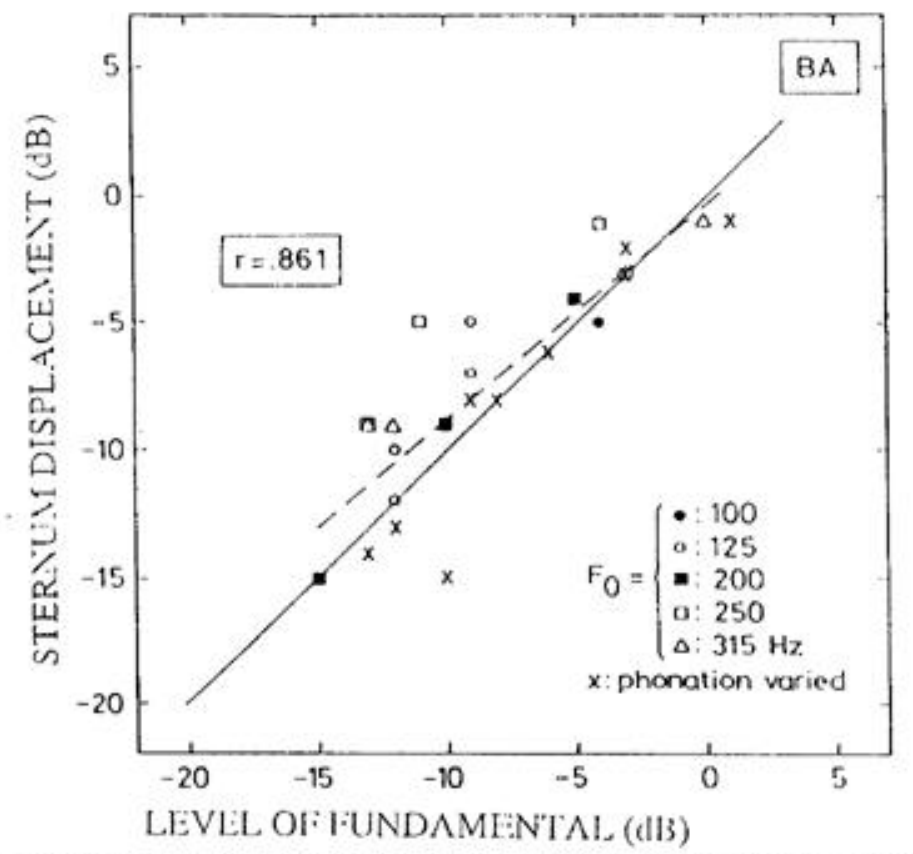

Fig. 13. Sternum displacement level as function of the level of the fundamental of a radiated vowel sound in a bass singer subject phonating in different manners, such that the level of the fundamental changed while the vowel was kept essentially constant. (From Sundberg, 1983)

Singers often resort to a type of phonation which we have termed "flow phonation". It is characterized by a rather dominant fundamental achieved by using a minimum degree of vocal fold adduction, just so much that the vocal folds barely make contact during the closed phase of the vibratory cycle. It seems that good singers tend to avoid pressed phonation and strive to stay within flow phonation. The timbre then becomes "round", "generous", voluminous and perhaps one would also like to describe the timbre as typically "singer-like". Particularly for high, loud tones the difference is great between singers' and nonsingers' phonation. Even under such conditions, singers usually 
manage to avoid a routine usage of a scream-like, pressed type of phonation characterized by a low amplitude of the fundamental. Nonsingers, on the other hand, mostly tend to increase phonatory press more and more, the louder they sing at high pitches.

There seem to be two reasons for singers to avoid pressed phonation. One is that pressed phonation appears to cause damage to the vocal folds. However, what the human body can support varies tremendously between individuals. Thus, a technique fitting perfectly well for one person may ruin another person's voice totally in a short time. Still, a habitual use of pressed phonation tends to produce vocal problems, enforcing the singer a visit to the laryngologist and, perhaps, a week or two of total silence.

The second reason is related to musical expression. A pressed phonation is very expressive. It sounds like an urgent cry for help. If a singer always resorts to this type of expression when singing high pitches loudly, problems arise as soon as the text does not fit the phonatory expression. In this sense, the habitual use of pressed phonation under conditions of high and loud imposes disastrous limitations on the singer's expressive dynamics.

Summarizing this digression on the voice source, we have seen that the scream-like pressed phonation and the singer-like flow phonation are characterized by a low and a high amplitude of the fundamental, respectively. We also have seen that the amplitude of the fundamental is reflected in the sternum vibrations, the only substantial vibrations which do not show a clear vowel dependence. Thus, one can imagine that a singer may profit from the sensation of sternum vibrations, provided he can feel them.

What vibrations are perceptible? According to Verrillo (forthcoming) the skin's sensitivity to frequency is not much to brag about. The smallest detectable frequency difference amounts to something in the vicinity of five semitones, while the corresponding value for the sense of hearing is fifty times smaller, about 10 cent or even less. Therefore, the singer has no use whatsoever for sensing phonatory vibrations in order to check the pitch.

The amplitude of vibration is more interesting. The vibration sensitivity of the skin, given in terms of the lowest perceptible vibration amplitude, is strongly dependent on the magnitude of the vibrating area. If we may assume that phonatory vibrations scatter over a wide area of the chest wall, the data obtained with big contactor sizes are relevant. For such contactors the smallest perceptible vibration amplitude remains constant in the bass and starts to drop somewhere between 40 and $100 \mathrm{~Hz}$, depending on the contactor size. At higher vibration frequencies, it decreases by about $9 \mathrm{~dB} /$ octave down to a minimum at $250-300 \mathrm{~Hz}$. A plausible threshold curve has been plotted in Fig. 12 together with the singers' vibration level data.

Verrillo's data indicate that the singers' phonatory sternum vibration levels lie just above the threshold amplitude and course parallel to the threshold curve from the bass and up to the vicinity of $300-400 \mathrm{~Hz}$. Here, the vibration amplitude curve continues to drop while the threshold curve makes a turn and start to increase. This means that singers can feel sternum vibrations up to these frequencies, i.e., to or into the pitch range of D4 to G4. The possibility to perceive vibrations of higher frequencies than this seems small for two reasons. One is that the sensory organ is insensitive, and the other is that the phonatory vibrations have so small amplitudes at so high frequencies. A consequence of this is that a singer would be unable to sense his singer's formant in terms of vibrations. Thus, the vibrations in this frequency range revealed by Sakakura \& Takahashi seem irrelevant as a cause of sensing vibrations. Likewise, it seems clear that the vibrations in the feet described by Frei must be far below the threshold, as they lie about $45 \mathrm{~dB}$ below the thyroid vibration amplitude.

As the sensitivity to vibrations decreases above about $300 \mathrm{~Hz}$, and as phonatory vibrations decrease with rising frequency, phonatory vibrations cannot be perceived at high pitches. A bass singer should be able to sense his sternum vibrations throughout his range. A tenor should be able to sense them for the lower and middle part of his range, the notes above the pitch of E4. An alto is not likely to sense sternum vibrations, except for her lowest notes. This may be the reason why the register used in the female voice for the lowest pitches is called "chest" register.

Some singing teachers do not regard sternum vibrations as useful for controlling tone production. One reason for this may be that these vibrations seem dependent on pitch for all singers except basses. A confounding factor may also be the idea that ideal tone production is identical for all singers, regardless of category and sex. This idea would reflect timbral similarities between different singers. But voice research has revealed that male and female singers apply entirely different 
strategies to reach their phonatory goals. Therefore, vibration sensations which are useless for a soprano may not necessarily be useless also for other singers.

We have found that the phonatory vibrations are too faint to be perceptible in the frequency range above $350 \mathrm{~Hz}$, approximately, i.e., the pitch of F4. Yet, all singers tend to speak about placing the tone. Does this mean that our initial assumption was wrong? Are there perhaps other physical correlates of placement than perceptible vibrations? Do the very high pressure oscillations in the body tissues affect blood circulation in such a way that the effect can be termed "placing"? At present, this question must be left unanswered awaiting future investigations.

\section{REFERENCES}

Fant, G. (1960): Acoustic Theory of Speech Production, Mouton, The Hague.

Fant, G., Nord, L., \& Branderud, P. (1976): "A note on the vocal tract wall impedance," STL-QPSR No. 4, pp. 13-20.

Frei, J. (1978): Stimme und Körper. Zur Wahrnehmung der eigene Stimme Ganzkörpervibrations, Salm Musik-Verlag, Bem.

Gramming, P. \& Sundberg, J. (1988): "Spectrum factors relevant to phonetogram measurement," J.Acoust.Soc.Am. 83, pp. 2352-2360.

Kirikae, J., Sato, T., Oshima, H., \& Nomoto, K. (1964): "Vibration of the body during phonation of vowels," Rev. de Laryngologie, Otologie, Rhinologie 85, pp. 317-345.

Kitzing. P. \& Löfqvist, A. (1975): "Subglottal and oral air pressures during phonation - preliminary investigation using a miniature transducer system," Med. \& Biol.Eng., Sept., pp. 644-648.

Miller, D. \& Schutte, H. (1984): "Characteristic patters of sub- and supraglottal pressure variations within the glottal cycle," pp. 70-75 in Transcripts of the Thirteenth Sump. Care of the Professional Voice I: Scientific papers, The Voice Foundation, New York.

Milutinovic, Z. (1988a): "Physiology of tissue vibrations in the phonatory apparatus," Acta Phon.Lat. X, pp. 223-229.

Milutinovic, Z. (1988b): "Analysis of tissue vibrations in the phonatory apparatus as applied in clinical practice," Acta Phon.Lat. X, pp. 237-242.

Pawlowski, Z., Pawloczyk, R., \& Kraska, Z. (1985): "Epiphysic vibrations of singers studied by holographic interferometry," pp. 37-60 in (A. Askenfelt, S. Felicetti, E. Jansson, \& J. Sundberg, eds.) Proc. Stockholm Music Acoustics Conf. 1983 (SMAC 83), Publ issued by the Royal Swedish Academy of Music, Stockholm.

Rasmussen, G. (1982): "Human body vibration exposure and its measurement," Brüel \& Kjar Techn.Rev. No 1, pp. 3-31.

Sakakura, A. \& Takahashi, H. (1988): "Body wall vibration in trained and untrained voices," pp. $391-401$ in (O Fujimura, ed.) Vocal Physiology: Voice Production, Mechanisms and Functions, Raven Press, New York.

Schutte, H. \& Miller, D. (1986): "The effect of F0/F1 coincidence in soprano high notes on pressure at the glottis," J.Phonetics 14, pp. 385-392.

Sonninen, A., Vilkman, E., Karppinen, H., \& Karppinen, V. (1986): "Holographic interferometry in assessing abdominal and chest wall vibrations during phonation," Paper 277(VP) at the XXth Congr. of Int.Ass.Logopedics and Phoniatrics, Fol.Phoniat. 38, p. 354(A).

Sundberg, J. (1983): "Chest wall vibrations in singers", J.Speech \& Hear.Res. 26, pp. 329-340.

Sundberg, J. (1987): The Science of the Singing Voice, N. Ill. Univ. Press, DeKalb,IL.

Verrillo, R. (forthcoming): "Vibration sensations in humans," to appear in (J. Sundberg \& R. Verrillo, guest eds.) Vibration and Contraction. Physiological Aspects of Music Performance, Music Perception 9:2, Winter 1991. 